\title{
High-field magnetization of dilute rare earths in yttrium
}

\author{
Touborg, P.; Høg, J.; Cock, G. J.; Roeland, L. W.
}

\section{Published in:}

Physical Review B

Link to article, DOI:

10.1103/PhysRevB.10.2952

Publication date:

1974

\section{Document Version}

Publisher's PDF, also known as Version of record

Link back to DTU Orbit

Citation (APA):

Touborg, P., Høg, J., Cock, G. J., \& Roeland, L. W. (1974). High-field magnetization of dilute rare earths in yttrium. Physical Review B, 10(7), 2952-2954. https://doi.org/10.1103/PhysRevB.10.2952

\section{General rights}

Copyright and moral rights for the publications made accessible in the public portal are retained by the authors and/or other copyright owners and it is a condition of accessing publications that users recognise and abide by the legal requirements associated with these rights.

- Users may download and print one copy of any publication from the public portal for the purpose of private study or research.

- You may not further distribute the material or use it for any profit-making activity or commercial gain

- You may freely distribute the URL identifying the publication in the public portal

If you believe that this document breaches copyright please contact us providing details, and we will remove access to the work immediately and investigate your claim. 


\title{
High-field magnetization of dilute rare earths in yttrium
}

\author{
P. Touborg and J. Høg \\ Department of Electrophysics, Technical University, 2800 Lyngby, Denmark \\ G. J. Cock and L. W. Roeland \\ Natuurkundig Laboratorium, University of Amsterdam, The Netherlands \\ (Received 3 June 1974)
}

\begin{abstract}
Magnetization measurements have been performed on single crystals of $\mathrm{Y}$ containing small amounts of $\mathrm{Tb}, \mathrm{Dy}$, or $\mathrm{Er}$ at $4.2 \mathrm{~K}$ in fields up to $295 \times 10^{5} \mathrm{~A} / \mathrm{m}(370 \mathrm{kOe})$. Crystal-field and molecular-field parameters obtained from measurements of the initial susceptibility versus temperature give a satisfactory quantitative account of the high-field magnetization. This includes characteristic features due to the crossing and mixing of crystal-field levels.
\end{abstract}

The magnetic properties of rare-earth metals and alloys are strongly influenced by the crystalline electric fields. Magnetization measurements performed at high magnetic fields, corresponding to Zeeman energies comparable with the crystal-field splittings, offer in principle, a powerful means of studying these crystal fields. However, in the pure rare-earth metals the exchange interactions are comparable with, or dominate, the crystal-field interactions, making observations of direct crystalfield levels effects in the magnetization impossible, ${ }^{1,2}$ or difficult to interpret. ${ }^{3,4}$ By dissolving the rare earths in the nonmagnetic rare-earth-like metals $\mathrm{Y}, \mathrm{Lu}$, and $\mathrm{Sc}$ the exchange interactions can be sufficiently diminished to allow the interpretation of the effects in the magnetization caused by the crystalline electric field alone. $\mathrm{Tb}, \mathrm{Dy}$, and $\mathrm{Er}$, measured in this work, form tripositive ions when dissolved in $\mathrm{Y}$ having ground-state multiplets with $J=6, \frac{15}{2}$, and $\frac{15}{2}$ respectively. These multiplets are split by the hexagonal crystal field and the magnetic field.

In this paper we wish to report on magnetization measurements in fields up to $295 \times 10^{5} \mathrm{~A} / \mathrm{m}(370$ kOe) on single crystals of $\mathrm{Y}-1.234-$ at. $\%-\mathrm{Tb}, \mathrm{Y}-$ 0.898-at.\%-Dy, Y-3.60-at.\%-Dy, and Y-0.993at.\%-Er. The hexagonal crystal fields in these alloys have been deduced from measurements of the initial susceptibility ${ }^{5}$ and the basal-plane anisotropy. ${ }^{6}$ The present experiments were performed at the University of Amsterdam's high-field magnet, ${ }^{7}$ in which high fields are produced by passing large currents through a copper coil cooled with liquid Ne. During the measurements at $4.2 \mathrm{~K}$ described here, the samples were immersed in liquid He.

The magnetic moments were measured by induction in a detector-coil system as the field was decreased in a staircase-like form. The duration of each step was on the order of $60 \mathrm{msec}$, which is long enough for eddy currents to decay. The inaccuracy, which is mainly due to the irreproducibility of the pickup of the main field in the detector-coil system, was reduced to $2 \times 10^{-5} \mathrm{~A} \mathrm{~m}^{2}$ by fitting the high-field measurements - through a term linear in the field-to low-field measurements up to $40 \times 10^{5}$ $\mathrm{A} / \mathrm{m}$. The latter measurements were performed on a $5-\mathrm{Hz}$ vibrating sample magnetometer (VSM) described earlier. ${ }^{5}$ The magnetic moment of the $Y$ was measured and subtracted. Magnetization curves at $4.2 \mathrm{~K}$ in the $b$ and $c$ directions of the hexagonal-close-packed structures are shown in Figs. 1-4. The absolute uncertainty for the point at the highest field on each isotherm is shown.

The Hamiltonian used for calculating the magnetization comprises the crystal field terms

$$
\mathcal{H C}_{\mathrm{CF}}=\mathrm{B}_{20} \mathrm{O}_{20}+\mathrm{B}_{40} \mathrm{O}_{40}+\mathrm{B}_{60} \mathrm{O}_{60}+\mathrm{B}_{66} \mathrm{O}_{66},
$$

and the effective Zeeman Hamiltonian

$$
\mathfrak{H}_{\mathbf{Z}}=g_{J} \mu_{B} J(\overrightarrow{\mathrm{H}}+\underline{\lambda} \cdot \overrightarrow{\mathbf{M}})
$$

which includes the small exchange contribution in the molecular-field model. The $B_{l m}$ 's are the crystal-field parameters obtained earlier, ${ }^{5,6}$ the $O_{l m}$ 's are the Stevens operators. ${ }^{8} g_{J}$ is the Landé factor, $\mu_{B}$ the Bohr magneton, $\vec{J}$ the angular momentum operator, $\vec{H}$ the magnetic field, $\lambda$ the molecular field tensor, and $\overrightarrow{\mathrm{M}}$ the magnetization. $\lambda$ contains two independent parameters $\lambda_{11}$ and $\lambda_{1}$. These depend on the concentration of the alloy and have, as well as the actual concentrations, been determined earlier from susceptibility measurements on the same samples. ${ }^{5,6}$ The full curves in Figs. 1-4 are calculated using the above Hamiltonian. The theoretical calculations fit the experimental data very well. Minor deviations are expected due both to ordering effects which are not well described by the molecular-field model and to effects arising from exchange interactions with the conduction electrons which have been neglected. Zero-field susceptibility measurements ${ }^{6}$ have shown no signs of ordering in $\mathrm{Y}-0.993-$ at.\%-Er at $4.2 \mathrm{~K}$, while $\mathrm{Y}-1.234-\mathrm{at} . \%-\mathrm{Tb}$ 


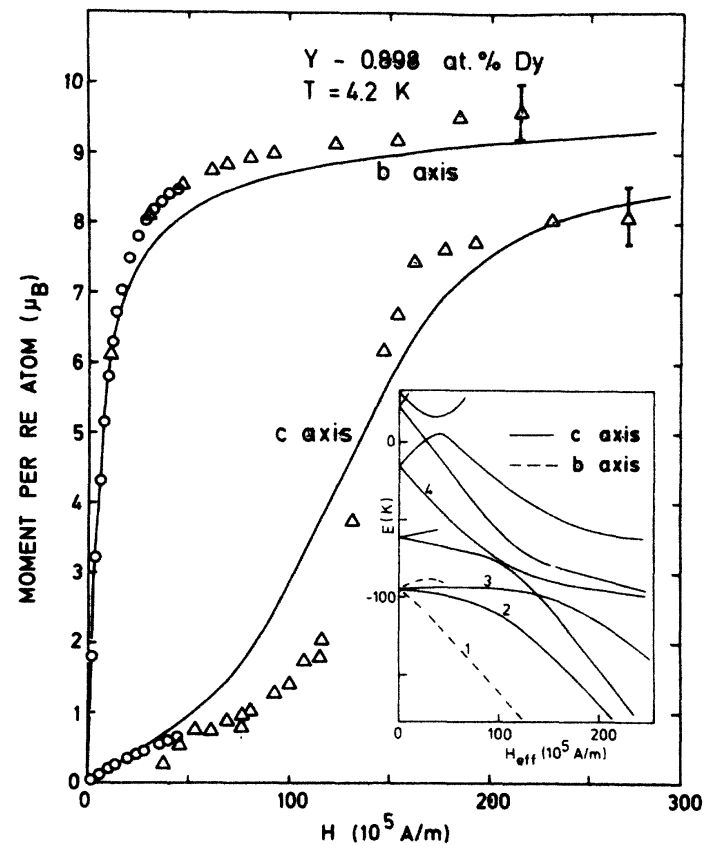

FIG. 1. Magnetization versus field for $\mathrm{Y}-0.898$-at. \%Dy. VSM data $O$, high-field data $\Delta$. The full curves are calculated using $B_{20}=0.336, B_{40}=-0.260 \times 10^{-2}, B_{60}$ $=0.335 \times 10^{-4}, B_{66}=-0.334 \times 10^{-3}$ (units of $\mathrm{K}$ ) and $\lambda_{1}=0.65$, $\lambda_{11}=-6.02$ [units of $10^{5}(\mathrm{~A} / \mathrm{m}) /\left(\mu_{B} /\right.$ atom $)$ ]. The energy levels are plotted against $\mathrm{H}_{\mathrm{oe}}$ in the inset.

shows features of incipient ordering. $Y-3.60-$ at.\%-Dy orders below $8 \mathrm{~K}$ in a helix ${ }^{9}$ with the moments lying in the basal plane and is included here to illustrate the effects of this ordering. Short-

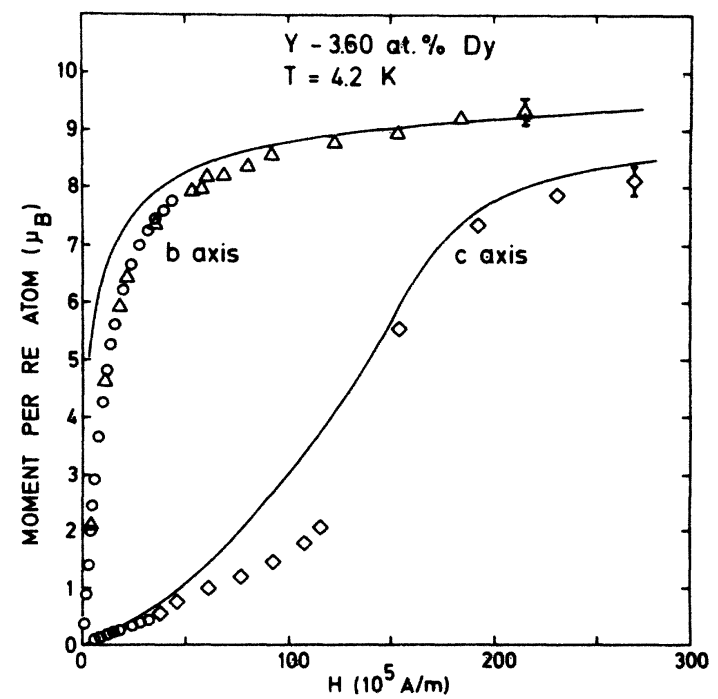

FIG. 2. Magnetization versus field for $Y-3.60$-at. \%Dy. VSM data 0 , high-field data $\Delta, 0$. The full curves are calculated using the $B_{l m}$ 's in Fig. 1 and $\lambda_{1}=1.0, \lambda_{11}$ $=-4.5$ [units of $10^{5}(\mathrm{~A} / \mathrm{m}) /\left(\mu_{B} /\right.$ atom $)$ ].

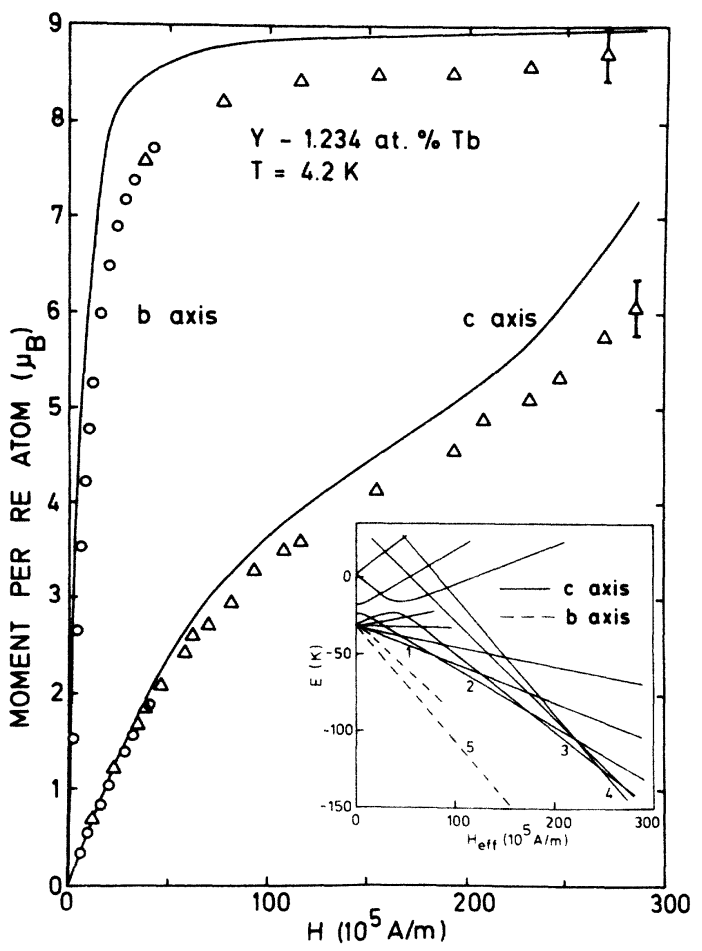

FIG. 3. Magnetization versus field for $Y-1.234-$ at. \%Tb. VSM data $\odot$, high-field data $\Delta$. The full curves are calculated using $B_{20}=0.945, B_{40}=0.589 \times 10^{-3}, B_{60}$ $=-0.145 \times 10^{-4}, B_{66}=0.100 \times 10^{-3}$ (units of $\mathrm{K}$ ) and $\lambda_{1}=0.15$, $\lambda_{11}=-5.97$ [units of $10^{5}(\mathrm{~A} / \mathrm{m}) /\left(\mu_{B} /\right.$ atom $)$ ]. The energy levels are plotted against $\mathrm{H}_{\text {opg }}$ in the inset.

range order effects in $\mathrm{Y}-0.898-$ at.\%-Dy are expected to be significant, and the deviations in the Dy alloys (Figs. 1 and 2) at lower fields in the $c$ direction, where the magnetization rises more sharply with the field than the calculations predict, are attributed to these effects.

To illustrate how the crystal fields account for the characteristic features of the magnetization curves, the positions of the crystal-field levels of importance are calculated from the Hamiltonian

$$
\mathfrak{H}=\mathcal{H}_{\mathrm{CF}}+g_{J} \mu_{B} \mathcal{J} \cdot \overrightarrow{\mathrm{H}}_{\text {off }}
$$

and plotted versus $\mathrm{H}_{\text {off }}$ in the figures. The magnetic moment is given in terms of the slope of the lowest populated levels according to the relation:

$$
\mu=\left(\sum_{i}-\frac{\partial \mathrm{E}_{i}}{\partial \mathrm{H}_{\text {off }}} e^{-\beta \mathrm{E}_{i}}\right) / \sum_{i} e^{-\beta E_{i}} .
$$

In zero field, the ground states of Dy (Fig. 1) form a Kramers doublet spanned by wave functions which are mainly $\left|J, J_{z}\right\rangle=\left|\frac{15}{2}, \pm \frac{1}{2}\right\rangle$. For fields in the $b$ direction, the large matrix element of $J_{X}$ within this doublet gives rise to the high moment at small fields. This is determined by the initial slope of the state 1, which for higher fields tends towards 


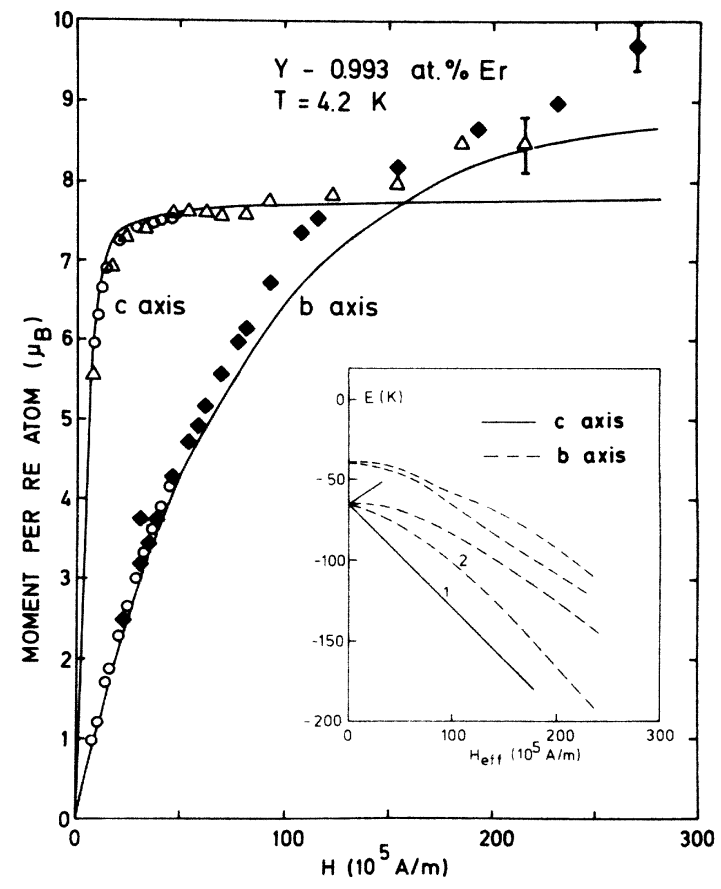

FIG. 4. Magnetization versus field for Y-0.993-at. \%Er. VSM data $O$, high-field data $\triangle$, $\diamond$. The full curves are calculated using $B_{20}=-0.309, B_{40}=0.600 \times 10^{-3}, B_{60}$ $=0.243 \times 10^{-4}, B_{66}=-0.279 \times 10^{-3}$ (units of K) and $\lambda_{1}=0.71$, $\lambda_{11}=-0.14$ [units of $10^{5}(\mathrm{~A} / \mathrm{m}) /\left(\mu_{B} /\right.$ atom $)$ ]. The energy levels are plotted against $\mathrm{H}_{\text {off }}$ in the inset.

$\left|J, J_{X}\right\rangle=\left|\frac{15}{2},-\frac{15}{2}\right\rangle$. When the magnetic field is applied in the $c$ direction, the ground-state doublet, 2 and 3 , gives rise to a small moment at lower fields. The level 4 is an approximate $\left|J, J_{Z}\right\rangle=\mid \frac{15}{2}$, $\left.-\frac{13}{2}\right\rangle$ state and is therefore rapidly lowered in the field. In the hexagonal crystal field, this level is mixed with the level 2 , thereby changing this from mainly $\left|J, J_{Z}\right\rangle=\left|\frac{15}{2},-\frac{1}{2}\right\rangle$ to mainly $\left|J, J_{Z}\right\rangle=\left|\frac{15}{2},-\frac{13}{2}\right\rangle$ at fields above $150 \times 10^{5} \mathrm{~A} / \mathrm{m}$. This accounts for the abrupt increase observed in the $c$-axis magnetization around this field.

The effects of helical ordering in $\mathrm{Y}-3.60-$ at. \%Dy, illustrated in Fig. 2, are large at low fields but insignificant at high fields. The discrepancies between theory and experiment are most pronounced in the basal-plane directions, presumably due to the fact that the moments order in this plane.

The crystal-field levels in $\mathrm{Tb}$ are highly accidentally degenerate in zero field (Fig. 3). For fields in the $c$ direction, several states cross through the ground state, resulting at $4.2 \mathrm{~K}$ in a smooth upward curvature at higher fields. This is clearly seen in the experimental data. The ground states $\left|J, J_{z}\right\rangle$ are, in order of development, the approximate states $1,|6,-2\rangle ; 2,|6,-3\rangle ; 3,|6,-4\rangle ;$ and 4 , $|6,-6\rangle$. For fields in the $b$ direction, mixtures of states create the state 5 , which is close to $\left|J, J_{X}\right\rangle$ $=|6,-6\rangle$.

In Er (Fig. 4) at zero field, the ground state is a Kramers doublet, which is spanned principally by $\left|J, J_{Z}\right\rangle=\left|\frac{15}{2}, \pm \frac{13}{2}\right\rangle$. When the field is in the $c$ direction, the ground state 1 has a large moment corresponding to $\left\langle J_{Z}\right\rangle \cong-\frac{13}{2}$. For increasing fields in the $b$ direction, the ground state 2 changes continuously towards $\left|J, J_{X}\right\rangle=\left|\frac{15}{2},-\frac{15}{2}\right\rangle$ due to mixing with higherlying states. This accounts for the crossing of the $b$-axis and $c$-axis magnetization curves.

In conclusion, measurements in fields with Zeeman energies comparable to the crystal-field splittings have proved to be an informative way of studying the crystal fields. The properties of dilute $\mathrm{Y}$-based rare-earth alloys are found to be accounted for by the crystal fields and the Zeeman interactions in which a small molecular field is included. Identical crystal-field and molecular-field parameters describe very well the magnetization both as a function of temperature ${ }^{5,6}$ and as a function of field, and also give a satisfactory account of the inelastic neutron scattering in an Y-Er alloy. ${ }^{10}$

The authors are grateful to Professor V. Frank and Professor A. R. Mackintosh for valuable suggestions during the course of this research. The high-field measurements form part of the research program of the "Stichting voor Fundamenteel Onderzoek der Materie" which is financially supported by the "Nederlandse Organisatie voor Zuiver Wetenschappelijk Onderzoek, " and also benefitted from financial support through the NATO Research Grant Program.
${ }^{1}$ G. J. Cock, L. W. Roeland, and P. Touborg (unpublished).

${ }^{2}$ J. J. Rhyne, S. Foner, E. J. McNiff, and R. Doclo, J. Appl. Phys. 39, 892 (1968).

${ }^{3}$ K. A. McEwen, G. J. Cock, L. W. Roeland, and A. R. Mackintosh, Phys. Rev. Lett. 30, 287 (1973).

${ }^{4}$ K. A. McEwen, P. Touborg, G. J. Cock, and L. W. Roeland, J. Phys. F (to be published).

${ }^{5} \mathrm{~J}$. Høg and P. Touborg, Phys. Rev. B $\underline{9}, 2920$ (1974).
${ }^{6} \mathrm{~J}$. Hog and P. Touborg, Phys. Rev. B (to be published).

${ }^{7}$ L. W. Roeland, F. A. Muller, and R. Gersdorf, Colloq. Int. Cent. Natl. Rech. Sci. 166, 175 (1967).

${ }^{8}$ K. W. H. Stevens, Proc. Phys. Soc. (London) A $\underline{65}, 209$ (1952).

${ }^{9} \mathrm{O}$. Rathmann (private communication on neutron diffraction experiments).

${ }^{10}$ O. Rathmann, J. Als-Nielsen, P. Bak, J. Hog, and P. Touborg, Phys. Rev. (to be published). 